\title{
Lagrangian Modelling of Fluid Sloshing in Moving Tanks
}

\author{
Eugeny Buldakov \\ UCL, Department of Civil Engineering, Gower Street, LONDON, WC1E 6BT, UK
}

\begin{abstract}
The paper considers the problem of sloshing of incompressible fluid in a moving 2-D rectangular tank under horizontal and vertical excitation. The problem is solved in Lagrangian variables by applying two approaches. First, a third-order asymptotic solution for resonant sloshing with a dominant mode is derived using a recursive technique. Then, fully nonlinear set of equations in the material coordinates is solved numerically by employing a finite difference method. Both methods are applied to a problem of high amplitude resonant Faraday waves and the obtained results are compared with experimental data known from the literature and a good agreement between the results of the two methods and the empirical data is demonstrated.
\end{abstract}

Keywords: Lagrangian formulation, asymptotic methods, numerical modelling, standing waves, sloshing in tanks

\section{Introduction}

Behaviour of fluid-containing structures is a challenging engineering problem essential for such diverse applications as liquid cargo transportation, dynamics of space vehicles, storage tanks or sloshing dampers. The unifying feature of all these problems is the presence of moving fluid considerably affecting dynamics of a coupled fluid-structure system. A crucial step in understanding behaviour of such systems is efficient description of high amplitude sloshing of fluid inside a moving container. This problem has received considerable attention for many years and numerous methods both analytical and numerical had been developed to address it.

Numerical models for sloshing are generally the same as for other water wave problems (Tsai and Yue, 1996; Scardovelli and Zaleski, 1999; Fenton, 
1999). The main difficulty in computing the solution of a fluid flowing with a free surface, is to devise a method to track efficiently the changing position of the free surface. The natural way of modelling deformation of a fluid domain is using equations of fluid motion in Lagrangian form, which to be solved in a fixed domain of Lagrangian labels. First works using finite-difference approximation of equations of fluid motion in Lagrangian formulation with applications to water wave problems appeared in early 70's. Brennen and Whitney (1970) used kinematic conditions of mass and vorticity conservation for internal points of a domain occupied by ideal fluid. Flow dynamics was determined by a free-surface dynamic condition. According to Fenton (1999) this approach apparently had not been followed. It seems that there are just a few works attempting using it (e.g. Nishimura and Takewaka, 1988). Hirt et al. (1970) used momentum equations in material coordinates for describing dynamics of viscous fluid. This formulation received relatively more attention from researchers. Later it was generalised for irregular triangular meshes (Fritts and Boris, 1979) and used for development of finite element models (e.g. Ramaswamy et al., 1986). This method however remains out of the mainstream and only occasionally appears in the literature (e.g. Radovitzky and Ortiz, 1998; Staroszczyk, 2009). Application of fully Lagrangian mesh models to viscous problems brings to a sharp focus their limitations. Boundary layers, wakes, vortices and other viscous effects lead to complicated deformations of fluid elements and large variations of physical coordinates over cells of a Lagrangian computational mesh. Resolving this problem led to development of Arbitrary Lagrangian-Eulerian (ALE) formulation (Hirt et al., 1974). ALE formulation uses a computational mesh moving arbitrarily within a computational domain to optimise shapes of computational elements and a problem is formulated in moving coordinates connected to the mesh. At certain regions of a computational domain the formulation can be reduced either to Eulerian (fixed mesh) or to fully Lagrangian (mesh moving with fluid) depending on problem requirements. This sophisticated method is capable of solving complicated problems with interfaces including surface waves and fluid-structure interaction. Detailed description of the ALE method, examples of application and comprehensive bibliography can be found in Souli and Benson (2013). ALE models are however complicated in both formulation and numerical realisation and are missing the main advantage expected from a Lagrangian method: simplicity of representing computational domains with moving boundaries. Simpler models can often give valuable results with less required resources. For ex- 
ample, many important water wave problems can be solved using ideal fluid model when deformation of elementary fluid volumes remains comparatively simple. These problems can be efficiently approached by much simpler Lagrangian models like one of Brennen and Whitney (1970). In this paper we introduce a fully Lagrangian numerical model which apart from keeping other advantages of the Lagrangian approach is extremely simple and can be optimised to achieve high computational efficiency.

Considerable contribution to understanding of behaviour of sloshing waves in tanks is due to analytical asymptotic models. These methods are based on an assumption that wave steepness is small and unknown functions are expanded into asymptotic series with respect to a small steepness parameter. In Eulerian description free surface boundary conditions are satisfied on a mean water level, which removes the complication of a moving boundary. Solution is usually looked for in the form of a Fourier series with respect to linear sloshing modes and higher modes usually contribute to higher terms of an asymptotic expansion. It is important to consider at least 3 terms of an asymptotic expansion to take into account cubic nonlinearity responsible for nonlinear corrections to a dispersion relation and crucially important for adequate description of long-term dynamics of a sloshing wave. For a comprehensive review of methods and results of asymptotic sloshing analysis readers are referred to Ibrahim (2005). We however should mention a notable contribution to development of modal sloshing theory made by Faltinsen and his colleagues presented in a series of papers on sloshing in 2-D rectangular tanks (Faltinsen et al., 2000), which was extended to three dimensions (Faltinsen et al., 2003) and later to circular (Faltinsen and Timokha, 2010) and spherical (Faltinsen and Timokha, 2013) basins. Recent developments of this approach include sloshing in tanks of complex geometry (Love and Tait, 2013) and tanks with perforated screens (Molin and Remy, 2013) with applications to tuned liquid dampers. Eulerian asymptotic models have significant limitations and application of an asymptotic technique in Lagrangian description can improve model behaviour for steep waves. There is an increasing interest to Lagrangian asymptotic wave models with applications to various problems including standing and sloshing waves (Shingareva and Celaya, 2007; Chen et al., 2009; Yang-Yih and Hung-Chu, 2009; Abrashkin and Bodunova, 2012). The general approach to Lagrangian asymptotic models is similar to the one for Eulerian ones. Asymptotic expansions are applied either directly to Lagrangian equations of fluid motion (Yang-Yih and Hung-Chu, 2009) or Eulerian expansions are transformed to 
Lagrangian ones (Chen et al., 2009). Modal representation is also a common feature of Lagrangian and Eulerian asymptotic approaches. However, unlike Eulerian models in Lagrangian asymptotic formulation free surface condition is satisfied on an exact free surface, which results in considerably better representation of surface shape. In this paper we present a third-order asymptotic model for resonant sloshing in a rectangular tank applying a recursive technique introduced in Buldakov et al. (2006). This approach leads to solutions exactly satisfying Lagrangian nonlinear continuity equation, which gives the new model some advantages compared to models using standard asymptotic expansions.

As a test case for models developed in this paper we use high amplitude standing waves in a rectangular tank generated by a parametric resonance due to vertical tank oscillations - the so called Faraday waves. Faraday waves received considerable interest through many years (e.g. Miles and Henderson, 1990) and are often used to test numerical and analytical models. Numerous experimental studies of the phenomenon are available in the published literature and in this work we use Jiang et al. (1998) and Bredmose et al. (2003) for qualitative and quantitative validation of the models. It should be emphasised that in the cases considered in the paper the tank moves with a prescribed frequency and amplitude, that is the tank and fluid are not a dynamically coupled system and the motion of each entity does not depend on the other.

The paper is structured as follows. Section 2 gives the mathematical formulation of the problem including equations of fluid motion in Lagrangian coordinates and boundary conditions. A recursive asymptotic solution for resonant sloshing with a dominant mode is derived in Section 3. A finitedifference scheme is introduced in Section 4, details of its numerical realisation and its accuracy are discussed. Section 5 defines computational cases and presents comparison of experimental and numerical results. Finally, Section 6

summarises paper achievements and discusses advantages and drawbacks of the presented methods.

\section{Problem formulation}

Let us consider a 2-D rectangular tank of length $b$ filled by an ideal incompressible fluid of depth $h$. The tank exhibits transitional motion in a laboratory coordinate system $(X, Z)$. A coordinate system $(x, z)$ is moving together with the tank with the horizontal axis $x$ pointing along the mean 
water level and the vertical axis $z$ pointing up along the left wall of the tank (Figure 1). We describe fluid motion in the tank using Lagrangian method by tracing marked fluid particles

$$
x=x(a, c, t) ; \quad z=z(a, c, t),
$$

where $(x, z)$ are coordinates of a particle marked by Lagrangian labels $(a, c)$ at time $t$. Lagrangian labels $(a, c)$ are used as a set of independent coordinates and $(x, y)$ are considered as unknown functions. Due to volume conservation for incompressible fluid the Jacobian $J$ of a mapping $(a, c) \rightarrow(x, z)$ is a motion invariant: $\partial J / \partial t=0$. This leads to the following Lagrangian form of the continuity equation

$$
J=\frac{\partial(x, z)}{\partial(a, c)}=J_{0}(a, c),
$$

where $J_{0}(a, c)$ is a given function of Lagrangian coordinates defined by initial positions of fluid particles associated with labels $(a, c)$.

Equations of motion of inviscid incompressible fluid in Lagrangian coordinates $(a, c)$ can be obtained using Hamilton's variational principle (e.g. Herivel, 1955). Let us represent the density of the Lagrangian in the following form

$$
\mathcal{L}=\mathcal{T}-\mathcal{U}+\rho P(a, c, t)\left(J-J_{0}(a, c)\right),
$$

where the kinematic continuity condition (1) is enforced by the Lagrange multiplier $P$, and $\rho$ is fluid density. The densities of the kinetic and potential energies of the fluid are

$$
\mathcal{T}=\rho\left(x_{t}^{2}+z_{t}^{2}\right) / 2 ; \quad \mathcal{U}=\rho g\left(1+Z^{\prime \prime}(t)\right) z+\rho \alpha \omega_{c}^{2} X^{\prime \prime}(t) x,
$$

where $X^{\prime \prime}(t)$ and $Z^{\prime \prime}(t)$ are Cartesian components of acceleration of a moving physical coordinate frame $(x, z)$. The vertical acceleration is scaled by the gravity acceleration $g$, and the horizontal one by a characteristic acceleration of fluid particles $\alpha \omega_{c}^{2}$, where $\alpha$ is a characteristic scale of particle displacement and $\omega_{c}$ is a characteristic frequency of wave motion. A practical choice of characteristic parameters depends on a particular problem. For a sloshing problem a typical wave amplitude can be chosen as $\alpha$ and a natural sloshing frequency as $\omega_{c}$. According to Hamilton's principle the variation of the action integral

$$
I=\int_{t_{1}}^{t_{2}} d t \iint_{D} \mathcal{L} d a d c
$$


is zero, where the integration takes place in the Lagrangian space over a domain $D$ occupied by the fluid. Taking the variation of (2) leads to the following equations describing dynamics of fluid inside $D$

$$
\begin{gathered}
x_{t t}+\frac{\partial(P, z)}{\partial(a, c)}+\alpha \omega_{c}^{2} X^{\prime \prime}(t)=0 ; \\
z_{t t}-\frac{\partial(P, x)}{\partial(a, c)}+g\left(1+Z^{\prime \prime}(t)\right)=0 .
\end{gathered}
$$

The Lagrange multiplier $P$ can be recognised as the ratio of pressure over density and the boundary condition on the free surface $c=0$ is $P=0$. These equations can be resolved with respect to the spatial pressure derivatives and rewritten in the following form

$$
\begin{aligned}
& \frac{\partial P}{\partial a}+g\left(1+Z^{\prime \prime}(t)\right) z_{a}+\alpha \omega_{c}^{2} X^{\prime \prime}(t) x_{a}=-x_{t t} x_{a}-z_{t t} z_{a} \\
& \frac{\partial P}{\partial c}+g\left(1+Z^{\prime \prime}(t)\right) z_{c}+\alpha \omega_{c}^{2} X^{\prime \prime}(t) x_{c}=-x_{t t} x_{c}-z_{t t} z_{c}
\end{aligned}
$$

The terms on the left hand sides of (3) are gradient components of a certain scalar function in the label space. Taking the curl of both sides of (3) we find that the value

$$
\Omega=\nabla_{a} \times\left(x_{t} x_{a}+z_{t} z_{a}, x_{t} x_{c}+z_{t} z_{c}\right)
$$

is a motion invariant: $\partial \Omega / \partial t=0$, where $\nabla_{a} \times$ is the curl operator in $(a, c)$ space. This gives the second kinematic condition in addition to (1)

$$
\Omega=\frac{\partial\left(x_{t}, x\right)}{\partial(a, c)}+\frac{\partial\left(z_{t}, z\right)}{\partial(a, c)}=\Omega_{0}(a, c)
$$

where $\Omega_{0}(a, c)$ is a given function. This is the Lagrangian form of vorticity conservation and for irrotational flow $\Omega=0$. Further detail on the Lagrangian form of the vorticity can be found in Casey and Naghdi (1991).

The Lagrangian formulation does not require a kinematic free-surface condition which is satisfied by specifying a fixed curve in Lagrangian coordinates corresponding to a free surface, e.g. $c=0$. The dynamics of the flow is described by a dynamic free-surface condition which can be obtained from the first equation in (3). For a case of constant pressure on the free surface $c=0$ we have

$$
x_{t t} x_{a}+z_{t t} z_{a}+g\left(1+Z^{\prime \prime}(t)\right) z_{a}+\left.\alpha \omega_{c}^{2} X^{\prime \prime}(t) x_{a}\right|_{c=0}=0 .
$$


The general formulation of the problem consists therefore of the continuity equation (1), the vorticity conservation equation (4), the free-surface condition (5) with suitable conditions on the bottom and side boundaries. Positions and velocities of fluid particles must be supplied as initial conditions.

One of the advantages of Lagrangian formulation is that a Lagrangian domain and an original correspondence between physical and Lagrangian coordinates is arbitrary and can be chosen from consideration of convenience of numerical or analytical analysis. It is convenient to use a rectangular Lagrangian domain which coincides with a physical domain occupied by still water

$$
0 \leq a \leq b ; \quad-h \leq c \leq 0 .
$$

The bottom and side boundary conditions can now be written as

$$
z(a,-h, t)=-h ; \quad x(0, c, t)=0 ; \quad x(b, c, t)=b .
$$

As initial condition we use stationary fluid with an initial surface perturbation

$$
\left.z\right|_{c=0 ; t=0}=\eta(x)
$$

where $\eta(x)$ defines an initial shape of the free surface in the physical domain. An initial horizontal coordinate of a fluid particle is used as the first Lagrangian label $a=\left.x\right|_{t=0}$ and the second Lagrangian label is uniformly distributed between fluid particles in a vertical column from the bed to the free surface: $c=h(z-\eta) /(h+\eta)$. The initial condition can now be written as

$$
\begin{gathered}
x(a, c, 0)=a ; \quad z(a, c, 0)=c \frac{h+\eta(a)}{h}+\eta(a) ; \\
x_{t}(a, c, 0)=0 ; \quad z_{t}(a, c, 0)=0 .
\end{gathered}
$$

The fist line of (7) can be used for calculating $J_{0}$ in (1). For the cases with horizontal excitation a surface perturbation is not required to initiate fluid motion and still water at equilibrium can be used as an initial condition. In this case (7) reduces to

$$
x(a, c, 0)=a ; x_{t}(a, c, 0)=0 ; z(a, c, 0)=c ; z_{t}(a, c, 0)=0,
$$

and the Jacobian $J=1$. 


\section{Asymptotic model}

Let us represent physical positions of fluid particles as

$$
x(a, c, t)=a+\alpha \xi(a, c, t) ; \quad z(a, c, t)=c+\alpha \zeta(a, c, t),
$$

where dimensionless functions $\xi$ and $\zeta$ describe particle displacement from the initial position $(a, c)$ and $\alpha$ is a characteristic scale of this displacement. We assume $\alpha$ to be small compared to the scale of a wave with a characteristic wave number $k$ and consider an asymptotic solution with $k \alpha \rightarrow 0$. Substitution to the continuity equation (1) gives in the leading approximation the classical Eulerian continuity equation for components of particle displacement

$$
\frac{\partial \xi}{\partial a}+\frac{\partial \zeta}{\partial c}=0
$$

In the second approximation the continuity can be satisfied by applying deformation of coordinates of the form

$$
a_{1}=a+\alpha \xi(a, c) / 2 ; \quad c_{1}=c+\alpha \zeta(a, c) / 2 .
$$

Further application of this deformation allows satisfying (1) at any order. Buldakov et al. (2006) demonstrated that the recursive representation

$$
\begin{aligned}
x(a, c, t)=a+\alpha \xi\left(a_{n}, c_{n}, t\right) ; & z(a, c, t)=c+\alpha \zeta\left(a_{n}, c_{n}, t\right) ; \\
a_{0}=a ; & c_{0}=c ; \\
a_{n}=a+\alpha \xi\left(a_{n-1}, c_{n-1}, t\right) / 2 ; & c_{n}=c+\alpha \zeta\left(a_{n-1}, c_{n-1}, t\right) / 2,
\end{aligned}
$$

satisfies the Lagrangian continuity equation (1) exactly if the recursion converges as $n \rightarrow \infty$ and asymptotically to the order $O(k \alpha)^{n+2}$ for a finite number of recursion steps. Functions $\xi$ and $\zeta$ satisfy (9) and can be represented using a single function $\Psi$ analogous to an Eulerian stream function

$$
\xi(a, c, t)=-\frac{1}{k} \frac{\partial \Psi(a, c, t)}{\partial c} ; \quad \zeta(a, c, t)=\frac{1}{k} \frac{\partial \Psi(a, c, t)}{\partial a} .
$$

To satisfy vorticity conservation (4) we represent function $\Psi$ as an asymptotic expansion for small $k \alpha$

$$
\Psi(a, c, t)=\Psi_{0}(a, c, t)+k \alpha \Psi_{1}(a, c, t)+(k \alpha)^{2} \Psi_{2}(a, c, t)+\cdots .
$$


In Buldakov et al. (2006) it is shown that function $\Psi_{0}$ satisfies the Laplace equation

$$
\nabla^{2} \Psi_{0}=0,
$$

and higher-order terms satisfy equations of the form

$$
\frac{\partial}{\partial t} \nabla^{2} \Psi_{n}=R_{n}(a, c, t)
$$

with right-hand sides depending on previous approximations. It is important to note that in spite of using asymptotic representation (12) for function $\Psi$ the Lagrangian continuity equation is satisfied exactly if the recursion converges since the recursive approximation is independent of a particular form of $\Psi$.

For a rectangular domain the leading-order solution can be expressed in the form of a Fourier expansion with respect to individual sloshing modes satisfying the Laplace equation in a rectangular domain and boundary conditions on the walls and bottom of the tank

$$
\Psi_{0}=\sum_{n=1}^{\infty} \frac{1}{n} \frac{\sinh \left(k_{n}(c+h)\right)}{\sinh \left(k_{n} h\right)} \sin \left(k_{n} a\right) F_{n}(t),
$$

where $k_{n}=\pi n / b$ is the wave number corresponding to the $n$-th sloshing mode. Substitution to the free-surface condition (5) gives in the first approximation the following equations for functions $F_{n}$ describing time evolution of individual modes

$$
F_{n}^{\prime \prime}+\omega_{n}^{2}\left(1+Z^{\prime \prime}(t)\right) F_{n}=\frac{2\left(1-(-1)^{n}\right)}{\pi} \frac{\omega_{c}^{2} X^{\prime \prime}(t)}{n B_{n}},
$$

where $\omega_{n}$ are natural frequencies satisfying linear dispersion relation $\omega_{n}^{2}=$ $g k_{n} \tanh \left(k_{n} h\right)$, and $B_{n}=1 / \tanh \left(k_{n} h\right)$. Equations (13) describing dynamics of linear modes are the same as the corresponding modal equations in Eulerian approach (e.g. Frandsen, 2004). Nevertheless, due to the essential nonlinearity of the recursive coordinates transformation certain nonlinear features can be captured even by a first-order solution. These, for example, are higher peaks and shallower troughs of a wave and existence of limiting wave amplitude when the recursive procedure starts diverging, which can be associated with wave breaking.

Right hand sides of equations for higher approximations include products of lower order solutions, which has to be expanded to Fourier series 
with respect to linear sloshing modes in order to obtain higher-order modal equations. This makes the analysis for high orders extremely difficult. To simplify the solution we consider sloshing with a single dominating mode, which generates only a limited number of modes at higher orders. Such an approach was used by Faltinsen et al. (2000) in their classical Eulerian analysis of nonlinear sloshing. Sloshing with a single dominating mode takes place when frequency of tank oscillations is close to a resonant frequency for this mode. For such oscillations a small amplitude of the horizontal excitation leads to high amplitude of a resonant mode, while amplitudes of all other modes remain small and are of the same order as excitation itself. Due to nonlinear mode-to-mode interaction the dominating mode generates modes with wave numbers which are integer multiples of a wave number of this mode. If amplitude of the dominating mode is small (but still much higher than the excitation amplitude) these nonlinear modes contribute to higher orders of approximation. Another way to generate a single dominating mode is trough mode instability due to a parametric resonance caused by vertical tank oscillations. Such oscillations with finite amplitude can lead to growing of a selected mode from a multimodal small initial surface perturbation.

Let us consider sloshing motion with a single dominating mode $m$. The corresponding wave number $k_{m}=\pi m / b$ is the natural choice for a characteristic wave number, and a steepness parameter $k_{m} \alpha$ is small $k_{m} \alpha \rightarrow 0$. We are looking for a third-order asymptotic solution $O\left(k_{m} \alpha\right)^{2}$, which includes cubical nonlinearity essential for capturing such a crucial effect as nonlinear detuning, which restricts amplitude growth during nonlinear resonance. A dominating mode is generated by small horizontal tank oscillations of order $O\left(k_{m} \alpha\right)^{2}$ at resonant or near-resonant frequency. Amplitude of vertical oscillations can be finite. We rewrite accelerations of the tank in the following form

$$
X^{\prime \prime}(t)=\left(k_{m} \alpha\right)^{2} A_{x}(t) ; \quad Z^{\prime \prime}(t)=A_{z}(t),
$$

where functions $A_{x}(t)$ and $A_{z}(t)$ remain finite as $k_{m} \alpha \rightarrow 0$. The dominating mode is the only mode contributing to the leading term of expansion (12). Due to nonlinear modal interaction it generates a mode $2 m$ contributing to the second term $\left(O\left(k_{m} \alpha\right)\right)$ and a mode $3 m$ contributing to the third term $\left(O\left(k_{m} \alpha\right)^{2}\right)$ of an expansion. Horizontal tank oscillations generate other modes, but being far from resonance they have amplitudes of the same order as oscillations $\left(O\left(k_{m} \alpha\right)^{2}\right)$ and contribute to the third term of (12). The 
solution can now be represented in the form

$$
\begin{aligned}
& \Psi=\sum_{n=1}^{3}\left(k_{m} \alpha\right)^{n-1} \frac{\sinh \left(n k_{m}(c+h)\right)}{n m \sinh \left(n k_{m} h\right)} \sin \left(n k_{m} a\right) F_{n m}(t) \\
& +\left(k_{m} \alpha\right)^{2} \frac{\sin \left(3 k_{m} a\right) \sinh \left(k_{m}(c+h)\right)+\sin \left(k_{m} a\right) \sinh \left(3 k_{m}(c+h)\right)}{4 \sinh \left(k_{m} h\right) \sinh \left(2 k_{m} h\right)} F_{m, 2 m}(t) \\
& +\left(k_{m} \alpha\right)^{2} \frac{\sin \left(3 k_{m} a\right) \sinh \left(k_{m}(c+h)\right)+\sin \left(k_{m} a\right) \sinh \left(3 k_{m}(c+h)\right)}{32 \sinh \left(k_{m} h\right)^{3}} F_{m, m, m}(t) \\
& +\left(k_{m} \alpha\right)^{2} \sum_{n \neq(1,2,3) m} \frac{\sinh \left(k_{n}(c+h)\right)}{n \sinh \left(k_{n} h\right)} \sin \left(k_{n} a\right) F_{n}(t)+O\left(k_{m} \alpha\right)^{3}
\end{aligned}
$$

where the first sum represents three nonlinearly interacting modes. Two following terms are used to satisfy the irrotationality condition (4). These terms modify the fluid kinematics and are not directly affected by the dynamic boundary condition. Their behaviour is defined by nonlinear modes through the following differential relations

$$
F_{m, 2 m}^{\prime}=F_{m}^{\prime} F_{2 m}-F_{m} F_{2 m}^{\prime} ; \quad F_{m, m, m}^{\prime}=-F_{m}^{\prime} F_{m}^{2}
$$

The final term in (14) represents non-interacting linear modes.

Functions $F_{n}$ describing time evolution of modes can be found from the free surface boundary condition (5). Substituting (14) back to (12), (10) and (5) we expand the resulting expression into a Taylor series for small $k_{m} \alpha$ up to the third order and collect terms proportional to $\sin (n k a)$ with the same $n$. Terms due to horizontal forcing are proportional to $A_{x}(t)$ and include coefficients $\cos (m k a), m=0,1,2 \ldots$, which should be expanded into a Fourier series with respect to $\sin (n k a), n=1,2 \ldots$ on the interval $a \in$ $[0, b]$. Finally we obtain the following system of three nonlinear differential 
equations describing time evolution of modal functions $F_{n}(t), n=m, 2 m, 3 m$

$$
\begin{aligned}
F_{m}^{\prime \prime}+\omega_{m}^{2}\left(1+A_{z}(t)\right) F_{m}=\left(k_{m} \alpha\right)^{2} \frac{4}{\pi} \frac{\omega_{c}^{2} A_{x}(t)}{m B_{m}} & \\
-\left(k_{m} \alpha\right)^{2} & \left(\frac{1}{4} \frac{3+B_{m}^{2}}{B_{m}}\left(F_{m}^{\prime \prime} F_{2 m}+F_{m} F_{2 m}^{\prime \prime}+F_{m}^{\prime} F_{2 m}^{\prime}\right)\right. \\
& +\frac{1}{8} \omega_{m}^{2}\left(1+A_{z}(t)\right) \frac{1+3 B_{m}^{2}}{B_{m}}\left(3 F_{m} F_{2 m}+F_{m, 2 m}\right) \\
& +\frac{1}{8}\left(9-B_{m}^{2}\right) F_{m} F_{m}^{\prime 2}+\frac{1}{16}\left(19-3 B_{m}^{2}\right) F_{m}^{2} F_{m}^{\prime \prime} \\
& \left.+\frac{1}{32} \omega_{m}^{2}\left(1+A_{z}(t)\right)\left(\left(5+7 B_{m}^{2}\right) F_{m}^{3}+\left(1+3 B_{m}^{2}\right) F_{m, m, m}\right)\right) ; \\
F_{2 m}^{\prime \prime}+\omega_{2 m}^{2}(1+ & \left.A_{z}(t)\right) F_{2 m}=-\frac{1-B_{m}^{2}}{B_{2 m}}\left(F_{m}^{\prime \prime} F_{m}+\frac{1}{2} F_{m}^{\prime 2}\right) \\
F_{3 m}^{\prime \prime}+\omega_{3 m}^{2}(1+ & \left.A_{z}(t)\right) F_{3 m}=\frac{4}{\pi} \frac{\omega_{c}^{2} A_{x}(t)}{3 m B_{3 m}} \\
& -\frac{3}{4} \frac{1-B_{m}^{2}}{B_{3 m}}\left(F_{m}^{\prime \prime} F_{2 m}+F_{m} F_{2 m}^{\prime \prime}+F_{m}^{\prime} F_{2 m}^{\prime}\right) \\
& -\frac{1}{8} \omega_{3 m}^{2}\left(1+A_{z}(t)\right) \frac{1-B_{m}^{2}}{B_{m}}\left(F_{m} F_{2 m}-3 F_{m, 2 m}\right) \\
& +\frac{3}{4} B_{m} \frac{1-B_{m}^{2}}{B_{3 m}}\left(F_{m}^{\prime \prime} F_{m}^{2}+F_{m}^{\prime 2} F_{m}\right) \\
& +\frac{1}{32} \omega_{3 m}^{2}\left(1+A_{z}(t)\right)\left(1-B_{m}^{2}\right)\left(F_{m}^{3}+3 F_{m, m, m}\right)
\end{aligned}
$$

Behaviour of linear modes for $n \neq m, 2 m, 3 m$ is described by equations (13).

To construct a solution we first solve an initial value problem including three nonlinear equations (16) for interacting modes, two equations (15) enforcing vorticity conservation to the required order and linear equations (13) for a desired number of non-interacting modes. Initial conditions for modal functions can be derived from initial shape and velocity of the free surface and for still water conditions all modal functions are zero together with their derivatives at $t=0$. Then, function $\Psi$ to be constructed using (14) and functions $\xi$ and $\zeta$ found using (11). Finally, physical positions of fluid particles $(x, z)$ can be calculated using the recursive procedure (10). A continuous solution is possible when recursion converges and regions of the Lagrangian domain where recursion diverges can be associated with wave breaking. 


\section{Finite difference numerical model}

We apply a finite difference technique for direct numerical solution of the problem $(1,4-7)$. Since equations $(1,4)$ for internal points of a computational domain include only first order spatial derivatives a compact four-point Keller box scheme (Keller, 1971) can be used for finite-difference approximation of these equations. For our selection of the Lagrangian computational domain the stencil box can be chosen with sides parallel to the axes of the Lagrangian coordinate system, which significantly simplifies the final numerical scheme. Values of unknown functions $x$ and $z$ on the sides of the stencil box are calculated as averages of values at adjacent points and then used to approximate derivatives across the box by first-order differences. The scheme provides the second-order approximation for the box central point. Time derivatives in (4) are approximated by second-order backward differences. It should be noted that the same scheme can be constructed by applying conservation of volume and circulation to elementary rectangular volumes (contours) with linear approximation of unknown functions on boundaries of elementary volumes.

Spatial derivatives in the free-surface boundary condition (5) are approximated by second-order central differences and special attention must be paid to approximation of second time derivatives which is crucial for the stability of the numerical scheme. In Buldakov (2013) it is demonstrated that using first-order backwards difference introduces numerical viscosity $O(\hat{\tau})$, where $\hat{\tau}=\sqrt{g k} \tau$ is the ratio of time step to a typical problem period. This leads to fast non-physical decay of perturbations. Third and higher-order approximations include terms with negative numerical viscosity leading to instability. We therefore use a second-order scheme which incorporates a numerical error to dispersion at the second order $O\left(\hat{\tau}^{2}\right)$ and weak dissipation at the third order $O\left(\hat{\tau}^{3}\right)$. The overall numerical scheme is of the second order in both time and space.

A fully-implicit time marching is applied, and Newton method is used on each time step to solve nonlinear algebraic difference equations. It is important to note that the scheme uses only 4 mesh points in the corners of the box for internal points of the fluid domain. Therefore, the resulting Jacobi matrix used by nonlinear Newton iterations has a sparse 4-diagonal structure and can be effectively inverted using specific algorithms, which are considerably faster and much less demanding for computational memory than general

algorithms of matrix inversion. The current version of the solver is using a 
standard NAG routine for inversion of general sparse matrices. To reduce calculation time inversion of a Jacobi matrix is performed at a first step of Newton iterations and if iterations start to diverge. Otherwise a previously calculated inverse Jacobi matrix is used. Usually only one matrix inversion per time step is required. An adaptive mesh is used in the horizontal direction with an algorithm based on the shape of the free surface in Lagrangian coordinates $z(a, 0, t)$ to refine the mesh at each time step in regions of high surface gradients and curvatures. A fixed non-uniform mesh is used in the vertical direction with higher mesh resolution near the free surface.

Convergence of the numerical scheme was studied on a problem of wave sloshing in a stationary tank. We use a square tank, which has the length of $b=0.7778 \mathrm{~m}$ and is filled with water of the same depth. The linear sloshing period of the first sloshing mode for this tank is $1 \mathrm{~s}$. A freely oscillating wave is exited using the initial surface perturbation $\eta(x, 0)=A_{0} \cos \left(k_{1} x\right)$, where $k_{1}=\pi / b$ is the wavenumber of the first sloshing mode and $A_{0}=0.05 \mathrm{~m}$ is the initial amplitude. The solution for 10 seconds of sloshing is calculated using different numbers of uniformly distributed mesh points and different time steps. The calculated shape of the free surface is expanded into a Fourier series with respect to sloshing modes and the behaviour of the first mode is analysed. Results of this analysis are presented in Figures 2 and 3. A linearised solution to the problem is the oscillation of the first mode with the period of $1 \mathrm{~s}$ and constant amplitude of $5 \mathrm{~cm}$. For the selected value of the initial amplitude the solution is weakly nonlinear. Nonlinear effects include generation of higher modes, energy exchange between modes and nonlinear corrections to sloshing frequencies. As can be seen from Figure 2 numerical errors include numerical dissipation and numerical corrections to the dispersion relation, which affects sloshing frequency. Figure 3 demonstrates that both these effects reduce and the solution converges with increasing number of mesh points and decreasing time step. For the main set of calculations presented in the paper a typical size of an adaptive computational mesh is $151 \times 31$ and a time step is $0.001 \mathrm{~s}$. About 8 seconds of computational time of a standard PC for each time step is required for these conditions. It should be mentioned that speed of calculations was not a priority and no solver optimisation was performed to increase it. 


\section{Results}

As a test case for the models presented above we use results of Bredmose et al. (2003) who performed an extensive experimental and numerical study of forced sloshing in a rectangular tank. In a series of experimental tests a $1.48 \mathrm{~m}$ long and $0.75 \mathrm{~m}$ high rectangular tank covered with a lid was oscillated horizontally and vertically and waves were observed for a range of water depth. A typical case includes horizontal oscillations at a linear resonant frequency of a third mode $\omega_{h}=\omega_{3}$. After approximately three periods, when the wave grows high enough, horizontal motion stops and the tank starts vertical oscillations with doubled frequency $\omega_{v}=2 \omega_{3}$. At this frequency the third mode is unstable and keeps growing due to parametric resonance resulting in steep breaking Faraday waves. Bredmose et al. (2003) also perform calculations by a Boussinesq model and a fully nonlinear boundary-integral solver and compare results with experiments. Further details of tank motion, experimental procedures and numerical methods can be found in the cited paper. In this study cases V05 and V21 are used with water depths $0.4 \mathrm{~m}$ and $0.302 \mathrm{~m}$, amplitudes of horizontal oscillations $0.5 \mathrm{~cm}$ and $1 \mathrm{~cm}$, amplitudes of vertical oscillations $3 \mathrm{~cm}$ and $1 \mathrm{~cm}$ and third linear sloshing frequencies $\omega_{3}=7.856 \mathrm{~s}^{-1}$ and $7.737 \mathrm{~s}^{-1}$ respectively.

First, we apply the fully nonlinear Lagrangian numerical model to a supplementary case of purely vertical oscillations with initial sinusoidal perturbation of the free surface, which was used by Bredmose et al. (2003) for testing the ability of the boundary integral solver to reproduce a 'table-top' waves observed in the experimental case V05. Figure 4 shows the result of the Lagrangian fully nonlinear model for lower (top) and higher (bottom) amplitude cases. The results of both methods are identical for the smaller amplitude case, which includes approximately one wave period. For the higher amplitude case the results coincide until $t=0.52 \mathrm{~s}$ when computation by the boundary integral solver breaks down. The Lagrangian solver is able to continue computations until $t=0.7 \mathrm{~s}$ to complete the wave period, but also breaks down shortly after. As can be observed in Figure 4, when the falling 'table-top' wave approaching the trough its extended edges are about to collide with the surface beneath. This process is characterised by extremely fast local deformations which cannot be modelled with reasonable number of mesh points and time step. Moreover, the collision itself is out of the scope of the current version of the Lagrangian solver and expected solution would include non-physical self-intersections of the free surface. 
There is no record of the exact tank motion available for the case V05, which differs in some details from the nominal motion described above. In addition, waves in this case touch the top of the tank, which cannot be simulated by the models. We therefore give only qualitative comparison of computational and experimental results for this case. A characteristic feature of wave motion observed in this experiment is a sharp-crested waves followed by a flat-topped one. Figure 5 demonstrates this feature in computational results by the Lagrangian fully nonlinear solver. The top figure shows wave evolution over half a period from $t=4.06 \mathrm{~s}$ to $t=4.50 \mathrm{~s}$ (Figure 5 , top). The downward motion starts with a sharp-crested wave which moves down forming a crushing crest. It creates a local distortion at the wave trough, which causes formation of a flat-topped wave on the following half-period (Figure 5, bottom). Miniature plunging breakers are forming on the sides of one of the rising flat-topped waves. This leads to solver breakdown shortly after $t=4.64 \mathrm{~s}$ due to excessively small temporal and spacial scales of the process. Similar to the previous case, calculations can continue after significant increasing of spatial mesh resolution and decreasing of time step, but the resulting solution will be non-physical because of self-intersection of the free surface. An occurrence of a flat-topped crest preceded by a sharp crest was observed experimentally by Jiang et al. (1998) who classified them as mode $\mathrm{A}$ and mode $\mathrm{B}$ waves respectively. Figure 5 accurately illustrates the process of wave transition from mode A to mode B presented in Jiang et al. (1998).

Figures 6 and 7 give comparison of results obtained by the Lagrangian asymptotic and fully nonlinear models. The solution of the asymptotic model includes 6 modes: three non-linearly interacting modes $n=3,6,9$ and three linear non-interacting modes $n=1,5,7$. The solution does not include linear modes with even $n$-s since they are not generated by horizontal excitation of the tank. One can hardly hope that a model using only a few first modes can capture small scale phenomena like those shown in Figure 5. However, Figure 6 demonstrates that predictions of the asymptotic model are in good agreement with the fully nonlinear one until the moment when local highly nonlinear phenomena start being important. As can be seen from Figure 7, the asymptotic model accurately predicts the entire wave profile for the case of a moderately steep wave. For a wave of high steepness it still gives the accurate profile shape for the considerable part of the free surface but makes a considerable error near the wave crest in the region of high deformation of fluid volume, where original model assumptions are far from being satisfied. 
Comparison of free-surface profiles calculated by both fully nonlinear and asymptotic Lagrangian methods with experimental results for the case V21 is presented in Figure 8. ${ }^{1}$ There is an excellent agreement between fully nonlinear Lagrangian solution and experiment until the finite-difference computations break down at $t=6.89 \mathrm{~s}$. The difference between experimental and computational profiles is practically withing line thickness. It is important to note the snapshots at $t=4.68 \mathrm{~s}$ and $t=5.12 \mathrm{~s}$, when the free surface is close to the mean water level. The shape of the surface at these times changes very fast and perfect agreement with the experiment here implies practically ideal representation of nonlinear dispersion by the numerical model. Bredmose et al. (2003) were unable to continue calculations by the boundary integral solver beyond time $t=6.83 \mathrm{~s}$ with high spatial resolution. Only a low resolution solution with local features being removed was obtained for larger times. The Lagrangian solver is also unable continue calculations far beyond this time. Figure 9 shows evolution of the free surface calculated by the Lagrangian solver over the last half of the wave period before the solver breaks down. A very thin falling jet can be observed on the right-hand wall of the tank. This is a process with considerably smaller time and length scales than typical scales of the entire wave, which requires unreasonable decrease of time step and increase of spatial mesh resolution to continue computations. It should be noted that this jet is not observed in the experiment. Moreover, no such jets ever appear at the tank walls for this experimental case and they exist only at internal crest positions $x / b=1 / 3$ and $x / b=2 / 3$. A feasible reason for suppressing wall jets in experiment is wall friction and effect of surface tension at a contact line between the free surface and the wall.

Figures 10 and 11 demonstrate that the Lagrangian asymptotic model gives very accurate predictions practically identical to predictions of the fully nonlinear solver for small and moderate wave amplitudes. After $t=4.5 \mathrm{~s}$ the asymptotic solution starts overpredicting surface elevation near wave crests. This error grows with increasing wave amplitude and eventually recursion stops converging at highest crests. However, apart from small regions around crests of highest waves, prediction of wave profiles is very good. Calculations

\footnotetext{
${ }^{1}$ The author is grateful to Dr Bredmose who kindly provided a routine calculating the exact tank motion for the case V21, which made detailed comparison of calculations with experiment possible.
} 
by the asymptotic model can be continued for times after the fully nonlinear solver breaks down and Figure 11 compares results of these calculations with experiment. The model does not resolve local features observed in the experiment such as falling jets. However, it is able to capture principal features of wave behaviour including both phase and general shape with high accuracy.

\section{Concluding remarks}

Conclusions are fairly obvious from the discussion above. In this section we are going to emphasise some strengths of the models, discuss their weaknesses and suggest possible ways of addressing them.

A considerable advantage of the fully nonlinear solver presented in the paper is its simplicity. Application of a finite-difference technique to approximate the problem $(1,4-7)$ is straightforward. Choice of a rectangular Lagrangian domain with lines of computational mesh parallel to axes of the Lagrangian coordinate system leads to a very compact numerical scheme. In most of the modern numerical water wave models using Lagrangian formulation this simplicity is overlooked. Applying complicated unstructured triangular meshes, like in Radovitzky and Ortiz (1998) significantly complicates a discrete formulation and reduces computational efficiency. This is justified for flows in complicated physical domains which cannot be continuously mapped to a rectangle or for flows with complicated vortical structure, but seems an unnecessary overcomplication for many water wave problems. Another advantage of the numerical model demonstrated in the paper is its exceptional accuracy in modelling of high amplitude non-breaking and near-breaking standing waves. The model is able to capture such features of nonlinear Faraday waves as falling jets and forming of flat topped waves. A crashing crest and a forming flat topped wave in calculated profiles (Figure 5) closely resemble similar features observed in experiments (Jiang et al., 1998; Bredmose et al., 2003). A falling jet (Figure 9) is qualitatively identical to those analysed by Longuet-Higgins and Dommermuth (2001) in their careful numerical study. Development of small-scale features of the free surface associated with high surface curvature and high acceleration eventually leads to calculations breakdown. Bredmose et al. (2003) use 5-point smoothing of the free surface which allows them to continue calculations of their boundary element solver to high amplitude breaking wave. All small-scale local features disappear from their solution, but the overall behaviour of the wave 
is represented with good accuracy. No smoothing was applied to results of calculations presented in this paper.

An essential step in constructing the Lagrangian asymptotic solution described in the paper is using a recursive procedure (10) to satisfy the continuity equation (1). This step allows to reduce the nonlinear Lagrangian continuity equation to a linear Eulerian form (9) and to express final solution via a single function similar to an Eulerian stream function. Vorticity conservation (4) and the free surface dynamic condition (5) are satisfied asymptotically, expanding the Lagrangian "stream function" into a conventional asymptotic series with respect to powers of a small wave steepness parameter. We would like to emphasise that in the case of recursion convergence the resulting solution satisfies the nonlinear Lagrangian continuity equation exactly regardless the order of the asymptotic expansion. This is a distinctive feature which differs this method from all other Lagrangian asymptotic solutions available in the literature. Direct numerical integration of the free surface gives conservation of fluid volume in the tank with the accuracy within the rounding error of computations. Stopping recursion after a few steps leads to a solution satisfying continuity asymptotically. For example, using two first steps of (10) leads to a solution similar to a standard expansion solution (e.g. Yang-Yih and Hung-Chu, 2009), which is formally of the same third order of approximation as a solution with converged recursion. However, such solution includes small time varying volume error which changes the total mass of fluid in the tank and can have negative effect when applying the model to simulate dynamics of coupled tank-structure systems.

An important question is the physical meaning of a solution when recursion diverges for some parts of the fluid domain. For free-surface flows physically meaningful solutions with surface discontinuities are possible, which are usually referred to as breaking waves. In Buldakov et al. (2006) it is shown that if recursion converges at some point of a fluid domain then a continuous solution exists in a vicinity of this point. This is a sufficient condition and recursion divergence does not mean that a continuous solution does not exist. One however could expect that solutions with diverging recursion are close to actual flow discontinuities and can be used for anticipation of possible wave breaking. For solutions considered in this paper recursion divergence is preceded by a cusp singularity at the crest of a wave. A cusp on a free surface is a natural feature of low order expansions in Lagrangian coordinates. It, for example, occurs at crests of a limiting Gerstner wave, which is the first term of an Lagrangian asymptotic expansion for a regular travelling wave. Such 
singularities can also be observed in both experimental (Jiang et al., 1998; Bredmose et al., 2003) and numerical (Longuet-Higgins and Dommermuth, 2001) studies of breaking standing waves. However, a following thin falling jet cannot be captured by a few terms of a Fourier expansion of a low-order Lagrangian asymptotic solution. Flat topped waves and micro breakers are also out of the scope of the model. Moreover, breaking predicted by recursion divergence does not coincide with real breaking events observed in the experiment. This is not surprising if we remember that the asymptotic model produces largest error near crests of high waves, where original assumptions of the model are far from being satisfied. Better modelling of these highly nonlinear features- if possible at all- requires constructing a higher-order asymptotic solution. Nevertheless, apart from considerable errors near crests of highest waves and inability to model small-scale features, the overall shape of the wave is represented by this simple model very well even for high amplitude breaking waves Another important advantage of the asymptotic model is its high speed of computations. For cases considered in this paper faster than real time modelling can be easily achieved using a standard PC. Together with fairly accurate results this makes the Lagrangian asymptotic model a good candidate for application to active sloshing control.

In general we can safely conclude that both the Lagrangian recursive asymptotic model and Lagrangian fully nonlinear solver demonstrated great potential in modelling high amplitude standing waves.

The author would like to thank Dr Bredmose and Cambridge University Press for their kind permission to use data from Bredmose et al. (2003) for models validation.

\section{References}

Abrashkin, A., Bodunova, Y., 2012. Nonlinear standing waves on the surface of a viscous fluid. Fluid Dynamics 47 (6), 725-734.

Bredmose, H., Brocchini, M., Peregrine, D. H., Thais, L., 2003. Experimental investigation and numerical modelling of steep forced water waves. Journal of Fluid Mechanics 490, 217-249.

Brennen, C., Whitney, A. K., 1970. Unsteady, free surface flows; solutions employing the Lagrangian description of the motion. In: 8th Symposium on Naval Hydrodynamics. Office of Naval Research, pp. 117-145. 
Buldakov, E., 2013. Tsunami generation by paddle motion and its interaction with a beach: Lagrangian modelling and experiment. Coastal Engineering 80, 83-94.

Buldakov, E. V., Eatock Taylor, R., Taylor, P. H., 2006. New asymptotic description of nonlinear water waves in Lagrangian coordinates. Journal of Fluid Mechanics 562, 431-444.

Casey, J., Naghdi, P., 1991. On the lagrangian description of vorticity. Archive for Rational Mechanics and Analysis 115 (1), 1-14.

Chen, Y.-Y., Hsu, H.-C., Hwung, H.-H., 2009. A Lagrangian asymptotic solution for finite-amplitude standing waves. Applied Mathematics and Computation 215 (8), 2891-2900.

Faltinsen, O., Rognebakke, O., Lukovsky, I., Timokha, A., 2000. Multidimensional modal analysis of nonlinear sloshing in a rectangular tank with finite water depth. Journal of Fluid Mechanics 407, 201-234.

Faltinsen, O., Rognebakke, O., Timokha, A., 2003. Resonant threedimensional nonlinear sloshing in a square-base basin. Journal of Fluid Mechanics 487, 1-42.

Faltinsen, O., Timokha, A., 2010. A multimodal method for liquid sloshing in a two-dimensional circular tank. Journal of Fluid Mechanics 665, 457-479.

Faltinsen, O., Timokha, A., 2013. Multimodal analysis of weakly nonlinear sloshing in a spherical tank. Journal of Fluid Mechanics 719, 129-164.

Fenton, J. D., 1999. Numerical methods for nonlinear waves. Advances in Coastal and Ocean Engineering 5, 241-324.

Frandsen, J. B., 2004. Sloshing motions in excited tanks. Journal of Computational Physics 196 (1), $53-87$.

Fritts, M., Boris, J., 1979. The Lagrangian solution of transient problems in hydrodynamics using a triangular mesh. Journal of Computational Physics 31 (2), 173-215.

Herivel, J. W., 1955. The derivation of the equations of motion of an ideal fluid by Hamilton's principle. Proceedings of the Cambridge Philosophical Society 51, 344-349. 
Hirt, C., Amsden, A., Cook, J., 1974. An arbitrary Lagrangian-Eulerian computing method for all flow speeds. Journal of Computational Physics $14(3), 227-253$.

Hirt, C., Cook, J., Butler, T., 1970. A Lagrangian method for calculating the dynamics of an incompressible fluid with free surface. Journal of Computational Physics 5 (1), 103 - 124.

Ibrahim, R. A., 2005. Liquid Sloshing Dynamics: Theory and Applications. Cambridge University Press, Cambridge.

Jiang, L., Perlin, M., Schultz, W. W., 1998. Period tripling and energy dissipation of breaking standing waves. Journal of Fluid Mechanics 369, 273299 .

Keller, H. B., 1971. A new difference scheme for parabolic problems. In: Numerical Solution of Partial Differential Equations, II (SYNSPADE 1970), Academic Press, New York, 327-350.

Longuet-Higgins, M., Dommermuth, D., 2001. On the breaking of standing waves by falling jets. Physics of Fluids 13 (6), 1652-1659.

Love, J. S., Tait, M. J., 2013. Nonlinear multimodal model for TLD of irregular tank geometry and small fluid depth. Journal of Fluids and Structures 43, 83-99.

Miles, J., Henderson, D., 1990. Parametrically forced surface waves. Annual Review of Fluid Mechanics 22 (1), 143-165.

Molin, B., Remy, F., 2013. Experimental and numerical study of the sloshing motion in a rectangular tank with a perforated screen. Journal of Fluids and Structures 43, 463-480.

Nishimura, H., Takewaka, S., 1988. Numerical analysis of two-dimensional wave motion using lagrangian description. Doboku Gakkai RombunHokokushu/Proceedings of the Japan Society of Civil Engineers 9 (5), 191-199, in Japanese.

Radovitzky, R., Ortiz, M., 1998. Lagrangian finite element analysis of newtonian fluid flows. International Journal for Numerical Methods in Engineering 43 (4), 607-619. 
Ramaswamy, B., Kawahara, M., Nakayama, T., 1986. Lagrangian finite element method for the analysis of two- dimensional sloshing problems. International Journal for Numerical Methods in Fluids 6 (9), 659-670.

Scardovelli, R., Zaleski, S., 1999. Direct numerical simulation of free-surface and interfacial flow. Annual Review of Fluid Mechanics 31 (1), 567-603.

Shingareva, I., Celaya, C., 2007. On frequency-amplitude dependences for surface and internal standing waves. Journal of Computational and Applied Mathematics 200 (2), 459-470.

Souli, M., Benson, D. J., 2013. Arbitrary Lagrangian Eulerian and FluidStructure Interaction: Numerical Simulation. Wiley-ISTE.

Staroszczyk, R., 2009. A Lagrangian finite element analysis of gravity waves in water of variable depth. Archives of Hydroengineering and Environmental Mechanics 56 (1-2), 43-61.

Tsai, W., Yue, D. K. P., 1996. Computation of nonlinear free-surface flows. Annual Review of Fluid Mechanics 28, 249-278.

Yang-Yih, C., Hung-Chu, H., 2009. A third-order asymptotic solution of nonlinear standing water waves in Lagrangian coordinates. Chinese Physics B 18 (3), 861-871. 

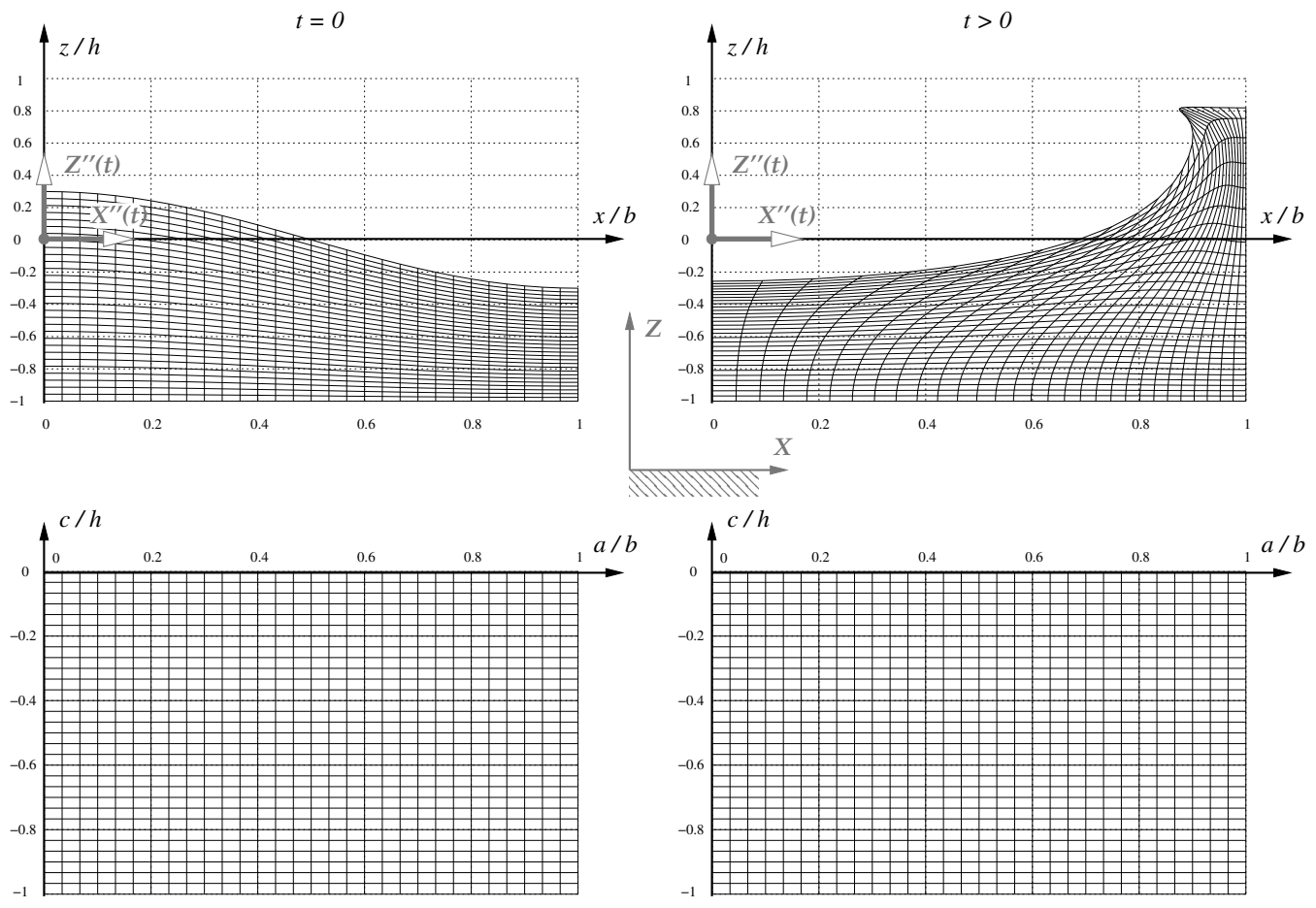

Figure 1: Coordinate systems and a fluid domain, an example with initial surface perturbation: $(X, Z)-$ a laboratory coordinate system; A physical coordinate system $(x, z)-$ is connected to a tank moving with accelerations $X^{\prime \prime}$ and $Z^{\prime \prime}$ and $(a, c)$ is a Lagrangian coordinate system. Left- initial conditions; right- developing flow. Top- a deforming physical fluid domain; bottom- a non-deforming Lagrangian fluid domain. 


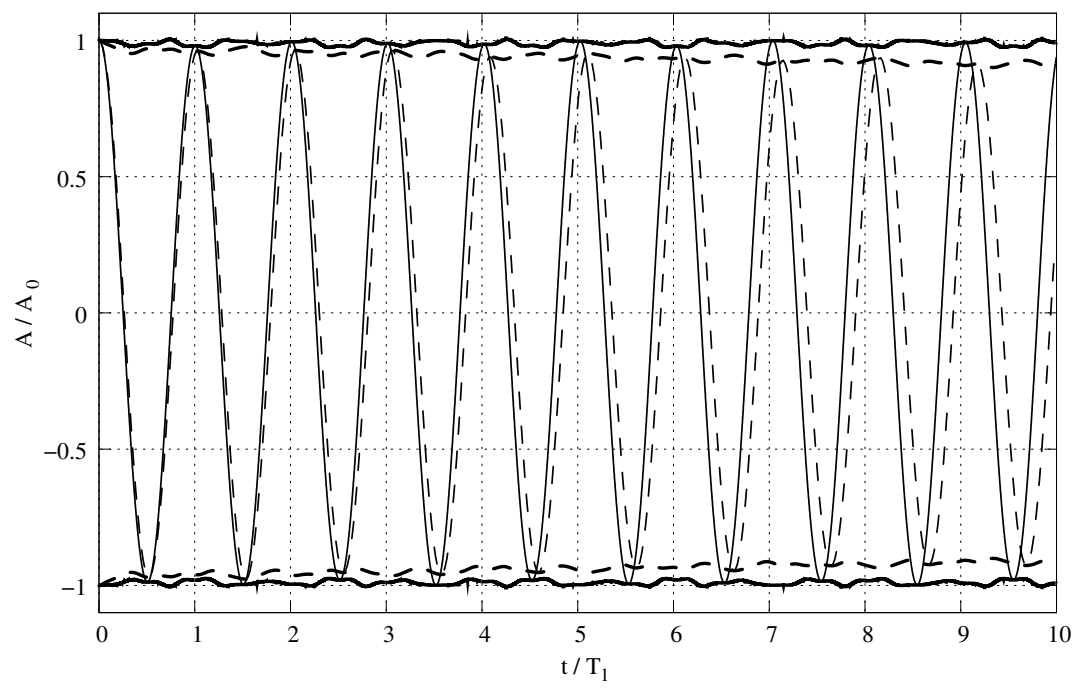

Figure 2: Convergence study. Behaviour of the first sloshing mode for different mesh sizes and time steps. Solid line: $51 \times 51, \delta t=0.001 \mathrm{~s}$. Dashed line: $11 \times 11, \delta t=0.02 \mathrm{~s}$. Thin lines: mode oscillation. Thick lines: slow change of mode amplitude. Time is scaled by the fist linear sloshing period. 

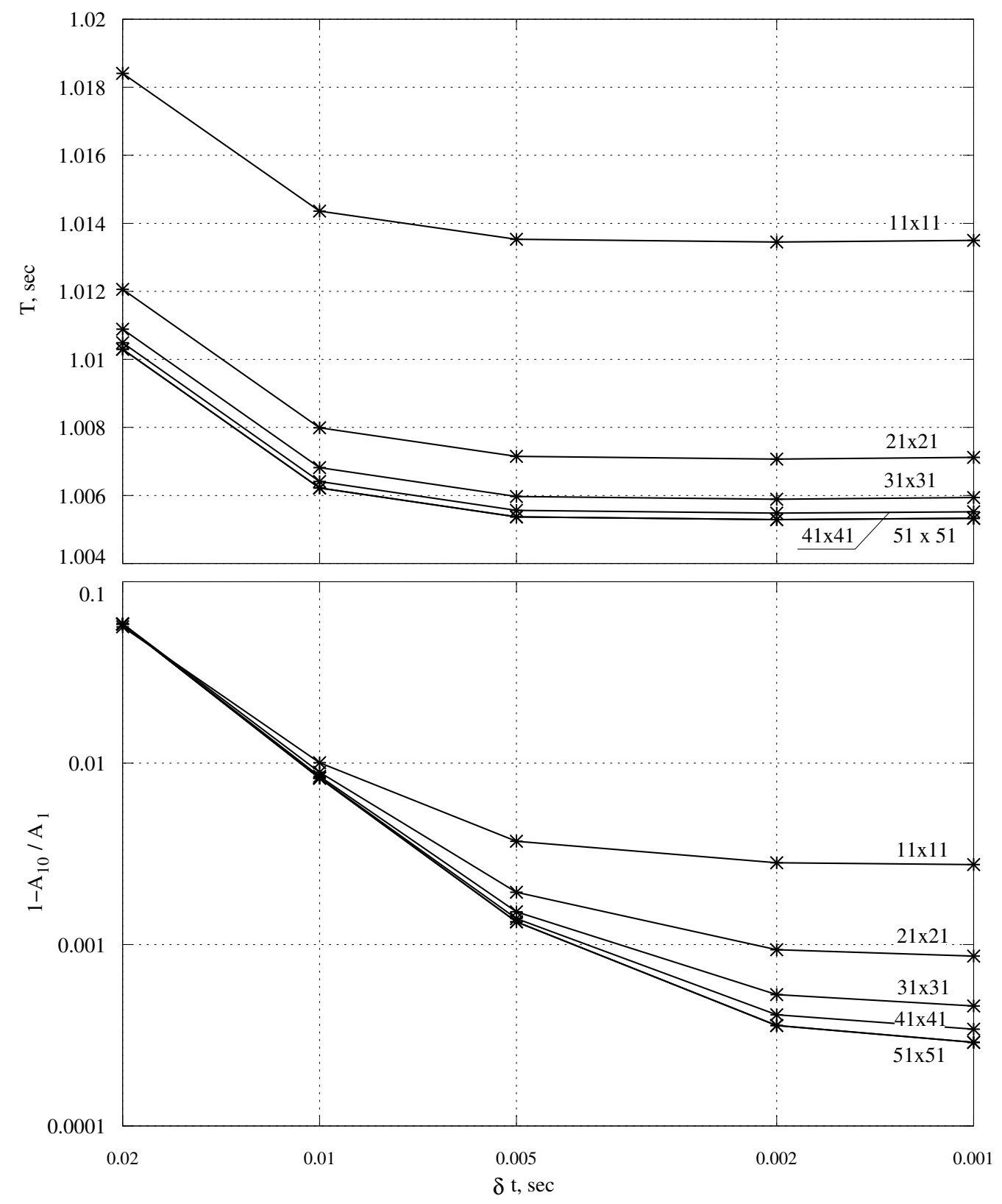

Figure 3: Convergence study. Period and numerical dissipation of the first sloshing mode for different mesh sizes $N_{a} \times N_{c}$ and time steps $\delta t$. Top: period of the first mode. Bottom: relative change of first mode amplitude between periods one $\left(A_{1}\right)$ and ten $\left(A_{10}\right)$. 

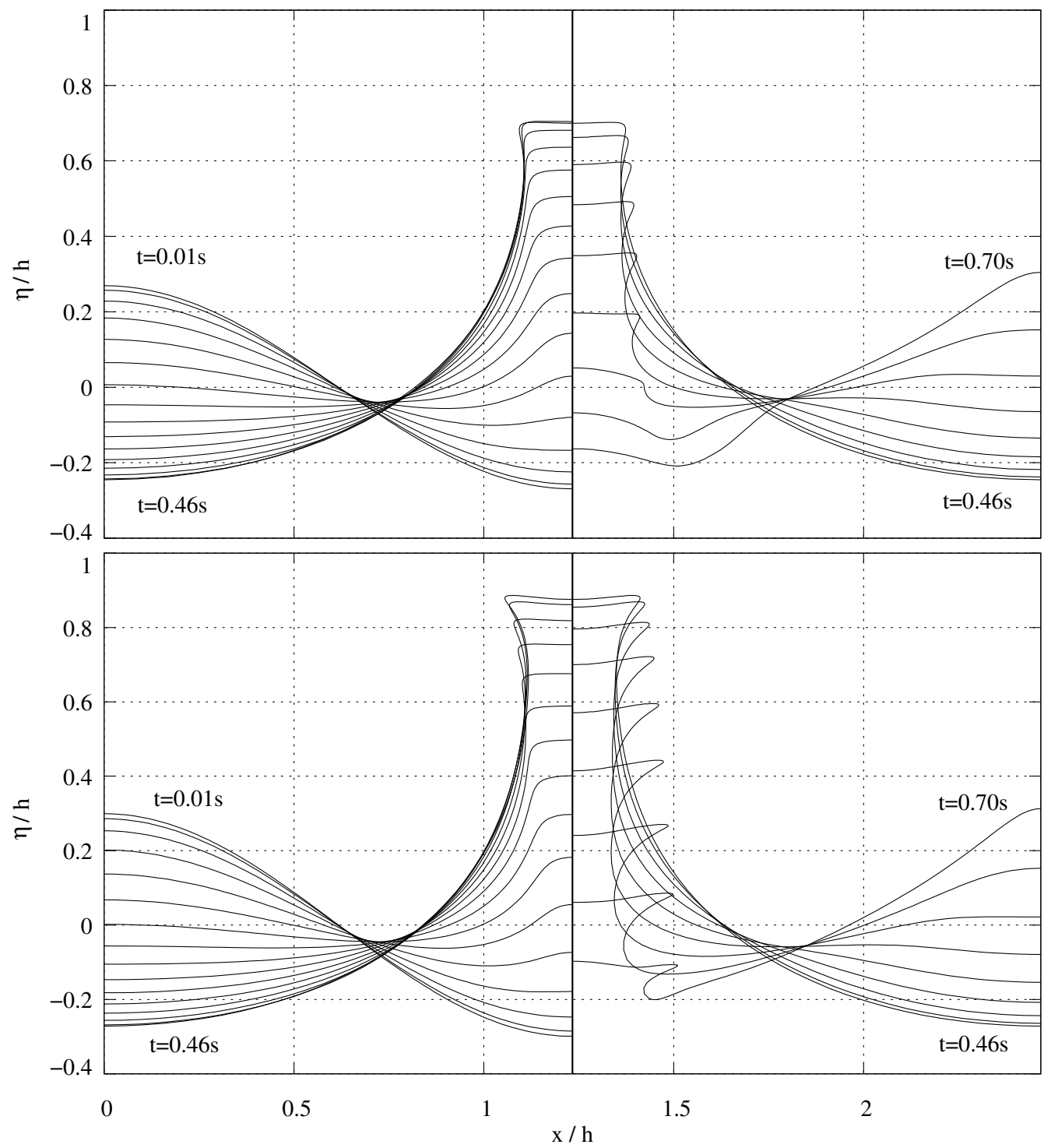

Figure 4: Evolution of the surface profile for the case V05 with vertical excitation only and initial sinusoidal surface perturbation calculated by the Lagrangian fully nonlinear solver. Left- upward motion; right- downward motion. Top- smaller excitation amplitude with smaller initial perturbation; bottom- larger excitation amplitude with larger initial perturbation. 

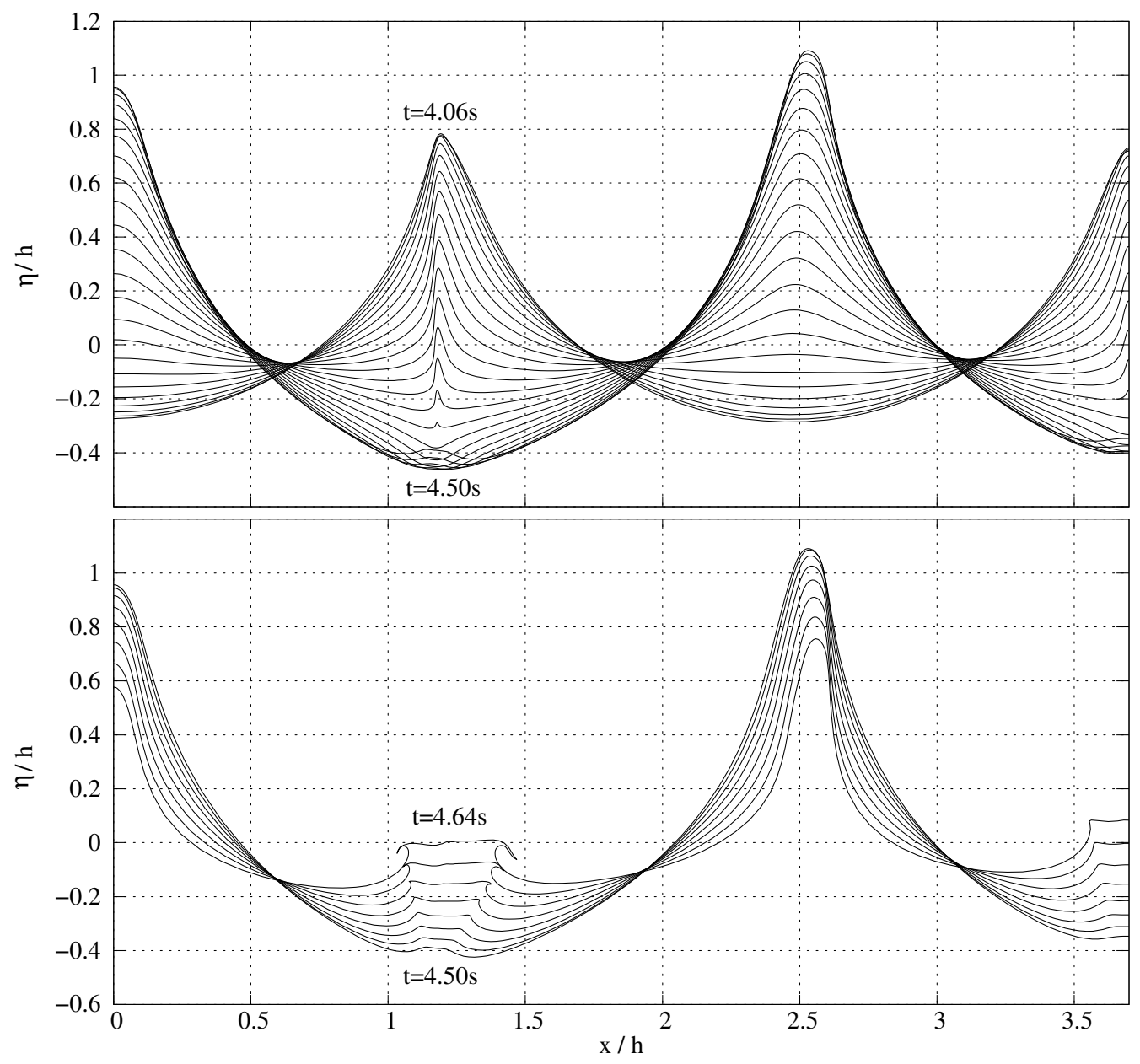

Figure 5: Evolution of the surface profile for the case V05 calculated by the Lagrangian fully nonlinear solver during a period when calculations break down. Top- downward motion; bottom- the initial stage of the upward motion. 


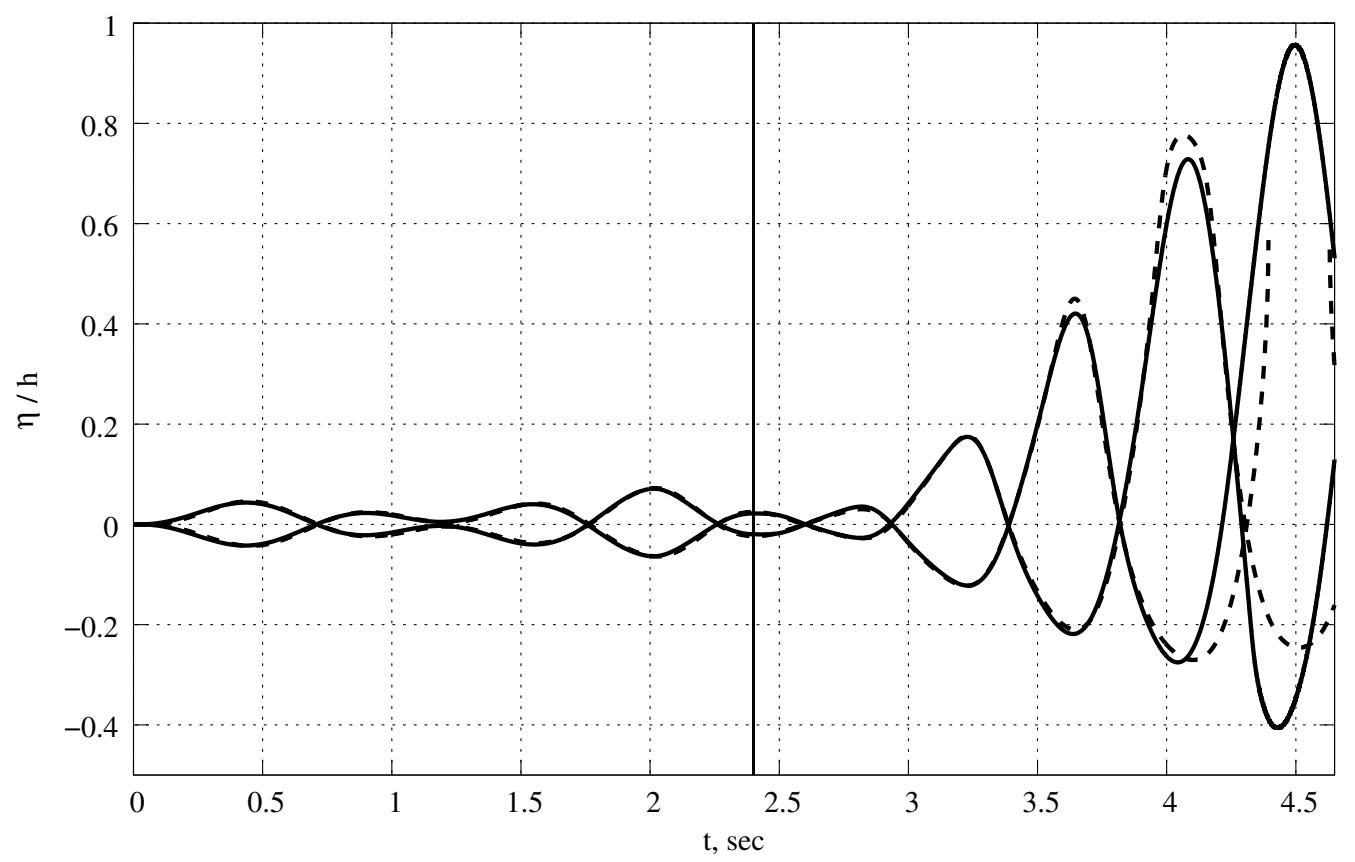

Figure 6: Time history of surface elevation at tank walls for the case V05. Solid line- Lagrangian fully nonlinear solver. Dashed line- Lagrangian recursive asymptotic model. The break of the dashed line represents recursion divergence. The thin vertical line corresponds to the time of starting vertical oscillations.

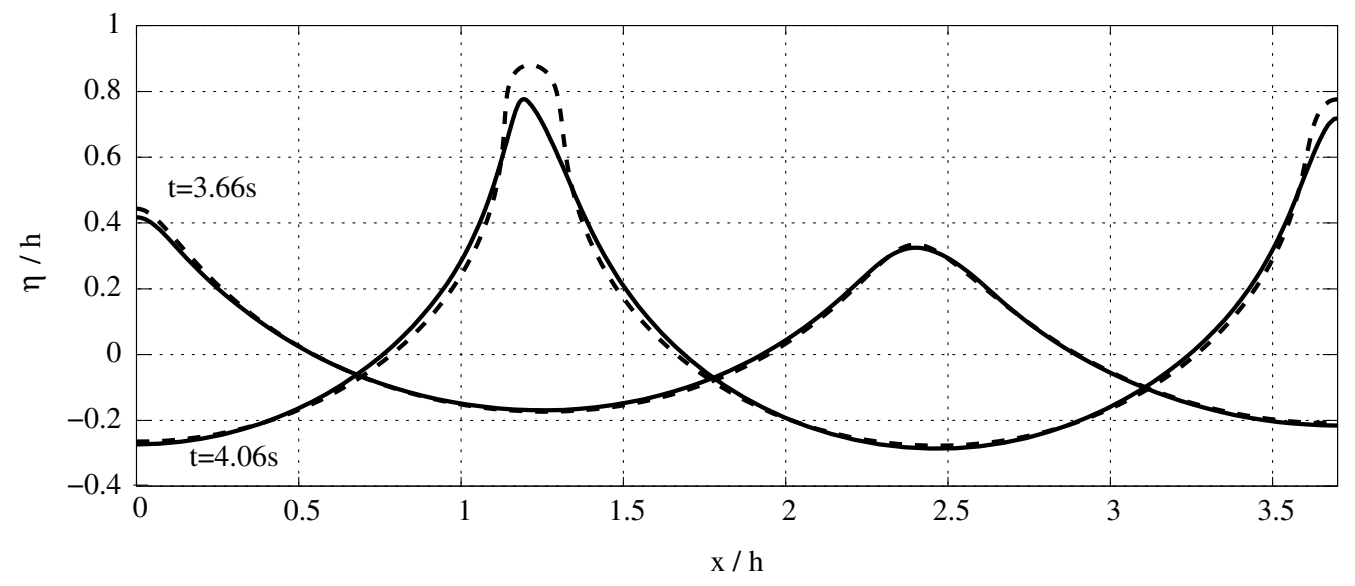

Figure 7: Surface profiles at selected times for the case V05. Solid line- Lagrangian fully nonlinear solver; dashed line- Lagrangian recursive asymptotic model. 


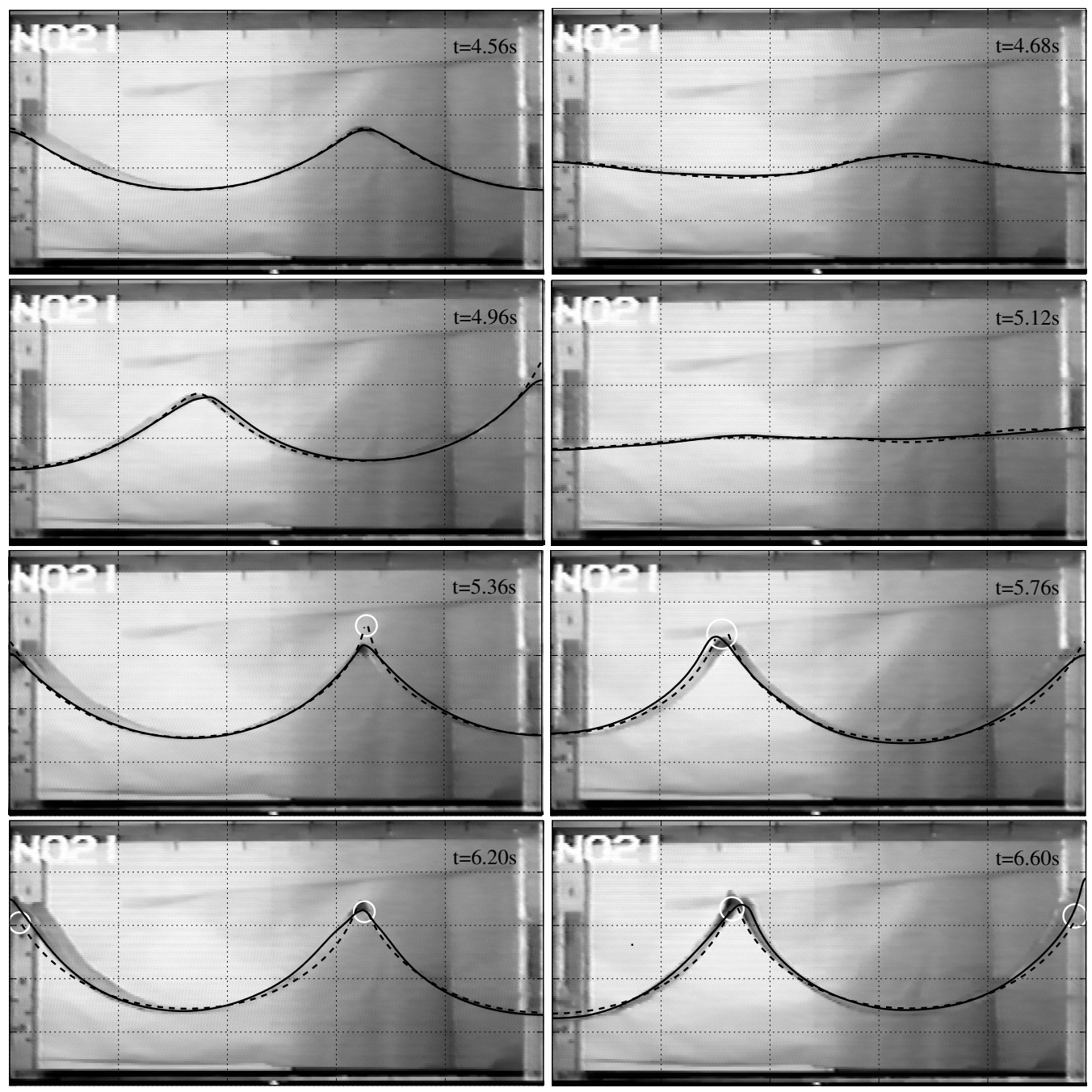

Figure 8: Comparison of surface profiles calculated by the Lagrangian fully nonlinear solver (solid) and Lagrangian recursive asymptotic model (dashed) with experimental results of Bredmose et al. (2003) (Case V21, represented by kind permission of the authors). 

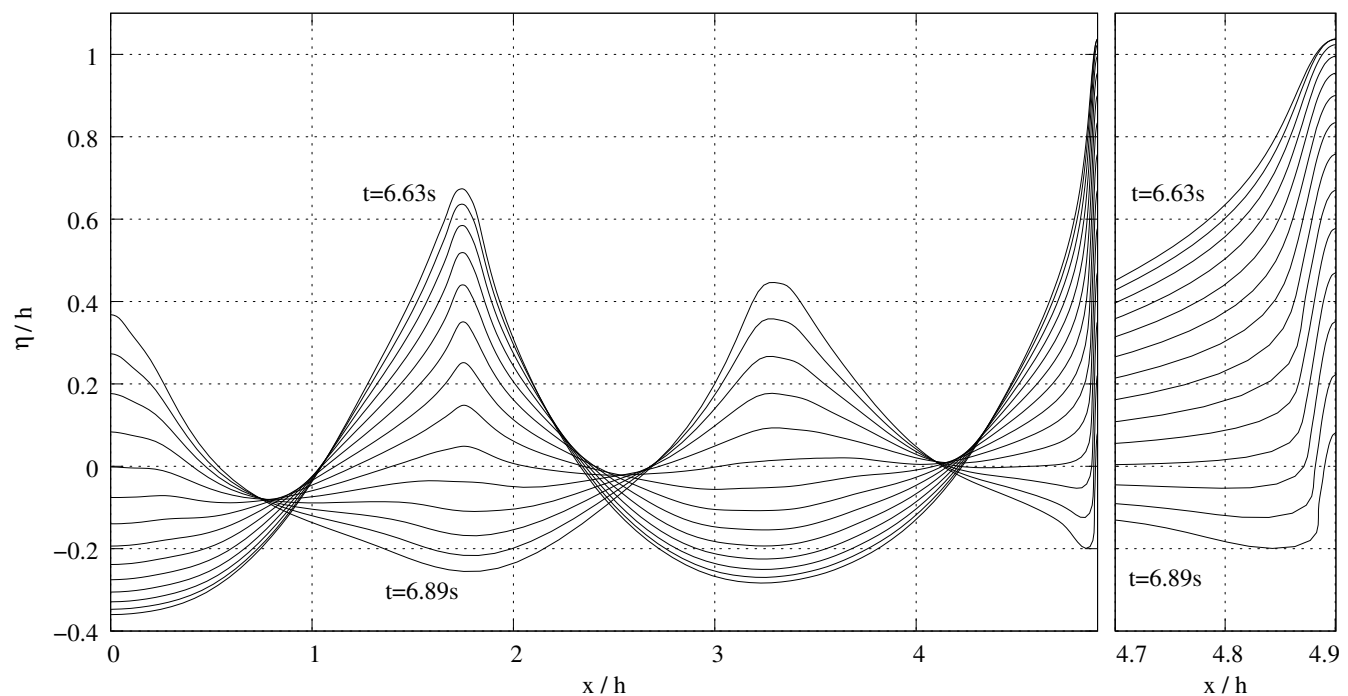

Figure 9: Evolution of the surface profile for case V21 calculated by the Lagrangian fully nonlinear solver before calculations break down. Right frame magnifies falling jet at the wall. 


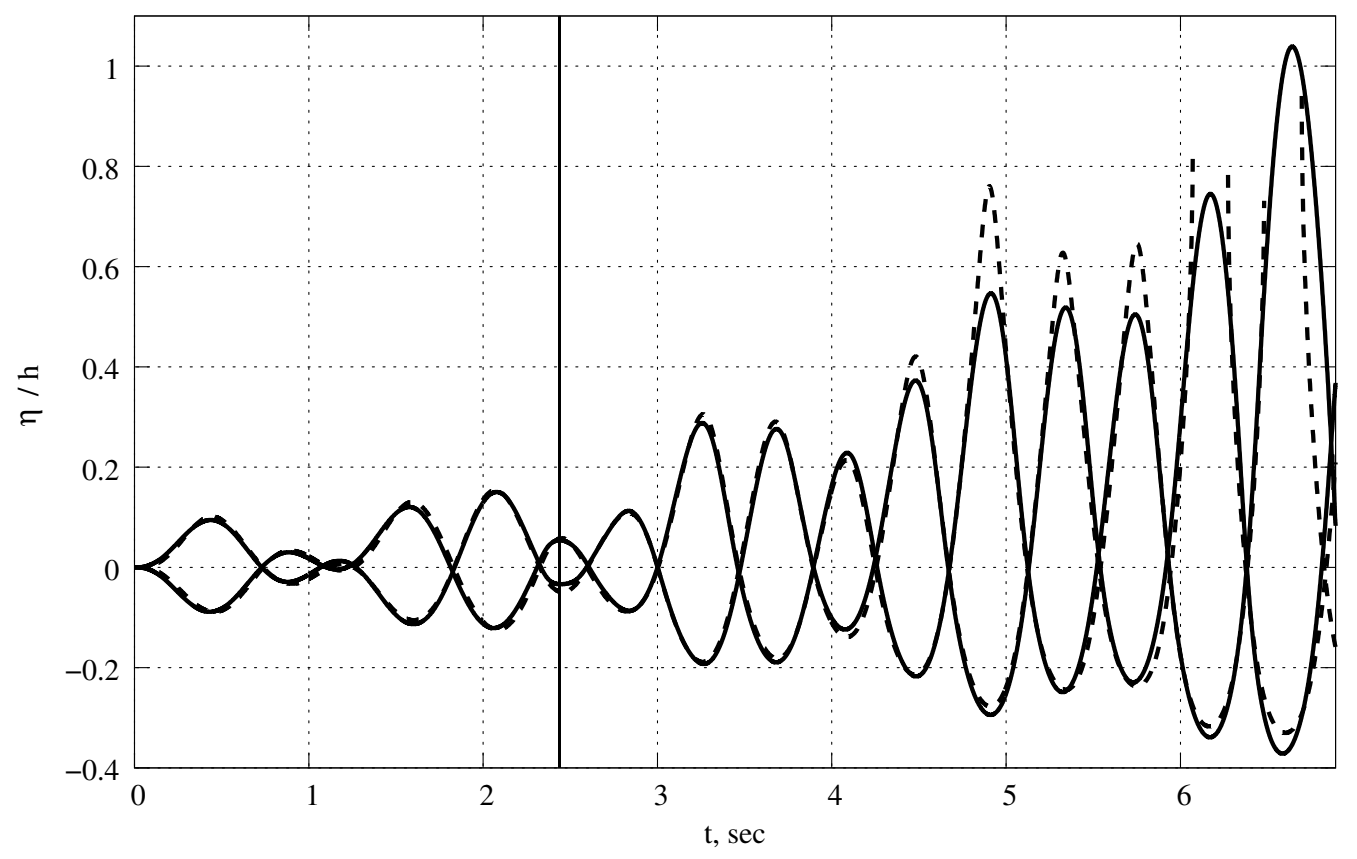

Figure 10: Time history of surface elevation at tank walls for the case V21. Solid line- Lagrangian fully nonlinear solver. Dashed line- Lagrangian recursive asymptotic model. The break of the dashed line represents recursion divergence. The thin vertical line corresponds to the time of starting vertical oscillations. 

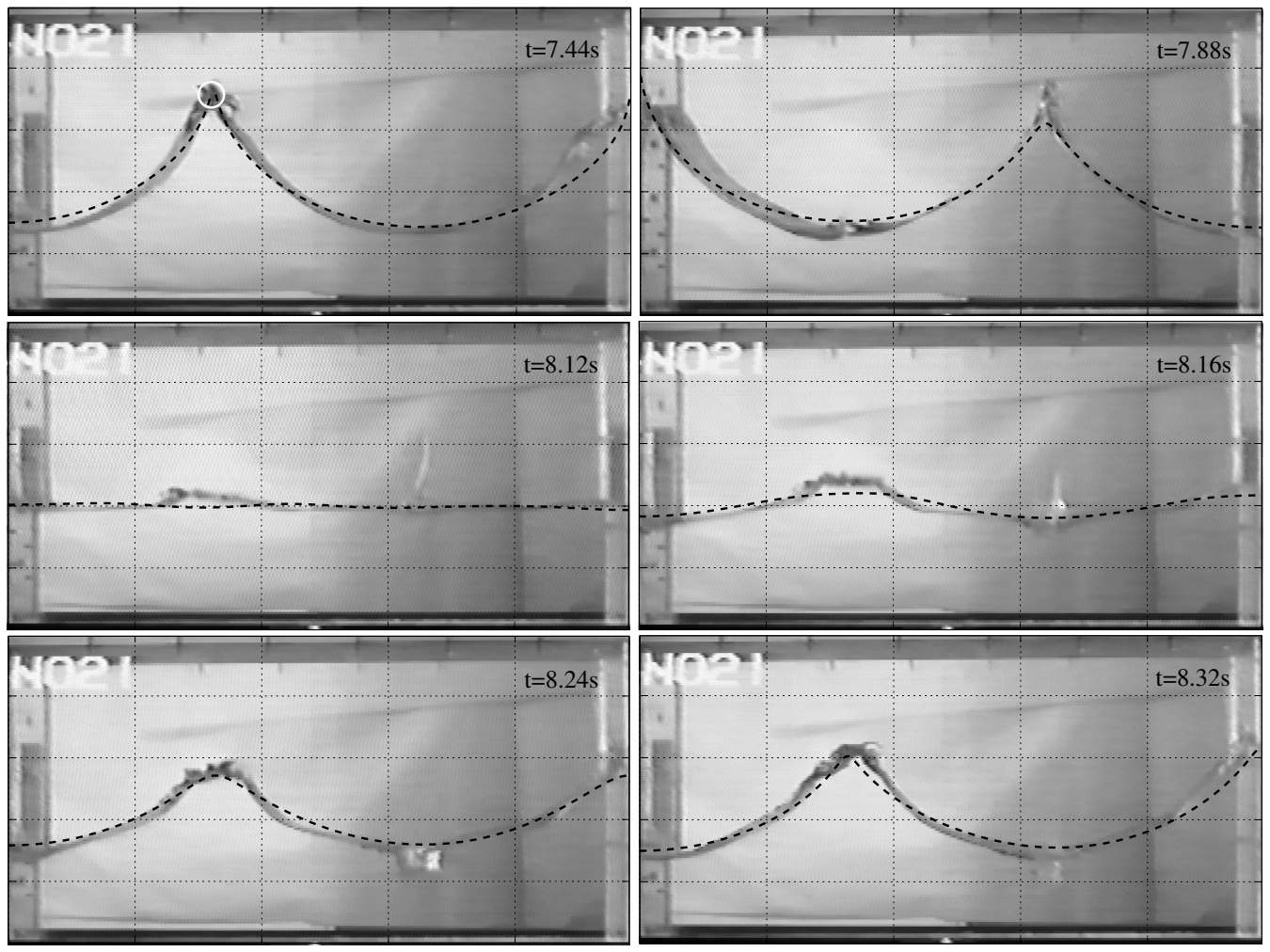

Figure 11: Comparison of surface profiles calculated by the Lagrangian recursive asymptotic model (dashed line) with experimental results of Bredmose et al. (2003) for large amplitude waves (Case V21, represented by kind permission of the authors). 